\title{
Características de la producción científica de la Revista INVI en la era SciELO, 2009-2016
}

\author{
Luis Campos Medina \\ César Orellana Mejías \\ Gustavo Carrasco Pérez
}

Instituto de la Vivienda, Facultad de Arquitectura y Urbanismo, Universidad de Chile - UCHILEFAU, Chile

ORIG INAL

\begin{abstract}
Resumen
En este artículo realizamos un análisis socio-demográfico y bibliométrico de Revista INVI, publicación periódica del Instituto de la Vivienda de la Universidad de Chile, basándonos en métodos propios y en la literatura disponible, con el propósito de identificar los niveles de productividad, citación e internacionalización de la revista, entre otros. El período seleccionado va entre los años 2009 y 2016, que corresponde al de su ingreso y permanencia en el índice SciELO, (Scientific electronic library online) que desde ya nos asegura indicadores internacionales de los grandes consorcios. El análisis pormenorizado de sus artículos originales, en base a indicadores de actividad, características de sus autores, referencias bibliográficas y algunos indicadores de impacto, entrega información valiosa para apreciar la evolución y el desarrollo específico de la producción científica en la Revista.
\end{abstract}

Palabras clave

Actividad científica; bibliometría; hábitat; índices

\section{Revista INVI: Characteristics of scientific production in SciELO age (2009-2016)}

\begin{abstract}
In this article we make a socio-demographic and bibliometric analysis of INVI Magazine, journal of the Institute of Housing at the University of Chile, based on own methods and on the available literature, in order to identify the levels of productivity, citation and internationalization of the journal, among others. The selected period is between 2009 and 2016, corresponding to their entry and permanence in the SciELO index (Scientific electronic library online), which now assures us international indicators of large consortia. The detailed analysis of the original articles based on indicators of activity, socio-demographic profile of the authors, references and some indicators of impact provides valuable information to appreciate the evolution and the specific development of the scientific production in the Journal.
\end{abstract}

Keywords

Bibliometrics; habitat; indices; scientific activity

\section{Introducción}

Revista INVI es una publicación del Instituto de la Vivienda de la Universidad de Chile creada en 1986, con el objetivo de difundir la labor desarrollada en el Instituto y posicionar la problemática de la vivienda social en el debate nacional chileno, tanto al interior de la academia, como fuera de ella. Se trata de una revista científica enfocada en los estudios sobre el hábitat humano y el territorio. En sus inicios, la revista fue un boletín, y tuvo como propósito difundir las inquietudes, la información y las necesidades del Instituto. Sin embargo, progresivamente fue creciendo en volumen y publicando artículos de índole científica. En el momento del cambio 
de siglo esta modificación en la revista era clara y lo fue aún más hacia fines de la primera década del segundo milenio, cuando se toma la decisión de posicionarse en los sistemas de indexación académica internacional.

En las páginas que siguen ofreceremos una aproximación analítica a través de indicadores bibliométricos con el propósito de caracterizar las formas que ha adoptado la producción de conocimiento científico en la revista. Hemos optado por observar esa producción desde distintas perspectivas, en virtud de la relevancia que ha adquirido factores como la producción colectiva de conocimiento, la internacionalización, la equidad de género, entre otros, en la discusión contemporánea sobre políticas científicas (Aguado-López, et. Al., 2009; CONICYT 2012, 2013). Pero también porque considerar estos factores hace posible observar las particularidades que adopta la producción de conocimiento en un dominio específico, en este caso, el de una revista que hace parte de los estudios territoriales y del hábitat.

\section{Materiales y métodos}

El estudio realizado es de carácter descriptivo y retrospectivo (Escorcia-Otálora y Poutou-Piñales, 2008). Los cálculos se han efectuado a partir del año 2009, año de incorporación de la revista a SciELO, lo que corresponde a 24 ediciones agrupadas en 8 volúmenes, dado el carácter cuatrimestral de la publicación (8 años, 3 números por año). Todo este material se obtuvo del sitio web de Revista INVI (http://revistainvi.uchile.cl/index.php/INVI) y de la colección física disponible en el Centro de Documentación del Instituto de la Vivienda. Junto con ello se optó por la consulta directa en el host de la versión electrónica de la revista a través de la Red SciELO, tomando como fecha de último procesamiento de sus artículos el día 12 de diciembre de 2016. También consultamos los datos de la Revista INVI en SCIMAGO, SCOPUS, REDALYC y WOS que, aunque recientes, son de relevancia pública.

De acuerdo a lo anterior, cabe señalar que esta revista está indexada actualmente en SCOPUS: Abstract and citation database of Elsevier B. V.; SciELO: Scientific Electronic Library Online; Scielo Citation Index, Thomson Reuters; Fuente Académica EBSCO, REDALYC, Red de Revistas Científicas de América Latina, el Caribe, España y Portugal, HAPI, Hispanic American Periodicals Index, DOAJ, Directory of Open Access Journals, REDIB, Red Iberoamericana de Innovación Conocimiento Científico, LATINDEX, Sistema Regional de Información en Línea para Revistas Científicas de América Latina, el Caribe, España y Portugal, PERIÓDICA, Índice de Revistas Latinoamericanas en Ciencias, PUBLINDEX y en el Sistema Nacional de Indexación de Publicaciones Científicas y Tecnológicas Colombianas, Colciencias. Falta, en consecuencia, el de la colección principal de Web of Science, actualmente en estado de postulación.

Nuestro análisis bibliométrico se circunscribe sólo a los 128 artículos originales del período, esto es, artículos inéditos, que presentan información propia segun los requerimientos de la discusión científica en el ámbito de los estudios territoriales, y que han sido sometidos a evaluación por pares (Camps, 2007). De cada trabajo original se ha extraído la siguiente información: Año/mes, volumen/número de la publicación, Título del artículo, palabras clave, nombre y apellidos de los autores, género de los autores, número de autores, profesión del primer autor, profesión de todos los autores, afiliación o adscripción institucional del y los autores, número de referencias bibliográficas, número de notas a pie de página, número de páginas. Para la introducción y análisis de los datos se ha utilizado el programa Excel 2016, no obstante, vale la pena señalar que el recuento se realizó de forma manual directamente desde las fuentes, efectuándose un minucioso proceso de curaduría de datos para facilitar el proceso de análisis. Los indicadores calculados fueron: número de artículos originales, índice de productividad (logaritmo del número de artículos originales), índice de colaboración e índice de referencias. A ellos agregamos información sobre las características de los autores (nacionalidad, género, y disciplina).

\section{Resultados}

\subsection{Tipo de artículo}

El volumen total de artículos originales publicados por Revista INVI en el período considerado (2009-2016) es de 128, lo que equivale al 52,67\% del período, algo más de la mitad de su producción editorial total (que también incluye opiniones, reseñas, separatas y editoriales) (Tabla $n^{\circ} 1$ ). Calculando la media de artículos originales por 
volumen publicado obtenemos 16 (128/8), siendo el promedio de artículos originales por número de 5,3 (128/24). Por su parte, la proporción de artículos originales respecto del volumen total publicado por número es de 1,9 $(243 / 128)$.

Tabla n¹: Total de artículos publicados en Revista INVI (2009-2016)

\begin{tabular}{|c|c|c|c|c|c|c|c|c|c|}
\hline Tipo de artículo & $\begin{array}{l}\text { Vol } 24 \\
(2009)\end{array}$ & $\begin{array}{l}\text { Vol } 25 \\
(2010)\end{array}$ & $\begin{array}{l}\text { Vol } 26 \\
(2011)\end{array}$ & $\begin{array}{l}\text { Vol } 27 \\
(2012)\end{array}$ & $\begin{array}{l}\text { Vol } 28 \\
(2013)\end{array}$ & $\begin{array}{l}\text { Vol } 29 \\
(2014)\end{array}$ & $\begin{array}{l}\text { Vol } 30 \\
(2015)\end{array}$ & $\begin{array}{l}\text { Vol } 31 \\
(2016)\end{array}$ & Total \\
\hline a) Artículo original & 12 & 13 & 15 & 20 & 16 & 18 & 15 & 19 & 128 \\
\hline b) Editorial & 3 & 3 & 3 & 3 & 3 & 3 & 3 & 3 & 24 \\
\hline c) Informativo & 6 & 5 & 7 & 8 & 7 & 7 & 5 & 10 & 55 \\
\hline d) Opinión & 2 & 2 & 2 & & 2 & 1 & 4 & 2 & 15 \\
\hline e) Reseña & 4 & 3 & 1 & 1 & 1 & & 1 & & 11 \\
\hline f) Separata & & 10 & & & & & & & 10 \\
\hline $\begin{array}{c}\text { Total Producción } \\
\text { Editorial }\end{array}$ & 27 & 36 & 28 & 32 & 29 & 29 & 28 & 34 & 243 \\
\hline
\end{tabular}

Fuente: Elaboración propia

\subsection{Tipo de autoría}

Considerando exclusivamente los 128 artículos originales, se obtuvo que el total de autores involucrados en este período es de 238. Acerca de este mismo punto, hay que consignar la autoría individual o colectiva de estos artículos originales, la que se distribuye de la siguiente forma: $48 \%$ de autoría individual (61 artículos) y 52\% de autoría colectiva (67 artículos), con 61 y 177 autores respectivamente (Gráfico n¹).

Comparados estos 3 elementos, vale decir, números de artículos, cantidad de autores y la relación entre autoría individual y autoría colectiva, por volumen, podemos inferir que el índice de colaboración, vale decir, el cociente entre número de firmas asociadas a cada uno de los trabajos y el número de los mismos (número de autores/número de artículos originales) publicados de Revista INVI, para los ocho años en estudio, es de 1,83 $(238 / 128)$.

\section{Gráfico n¹: Autoría Individual o Colectiva de Revista INVI (2009-2016)}

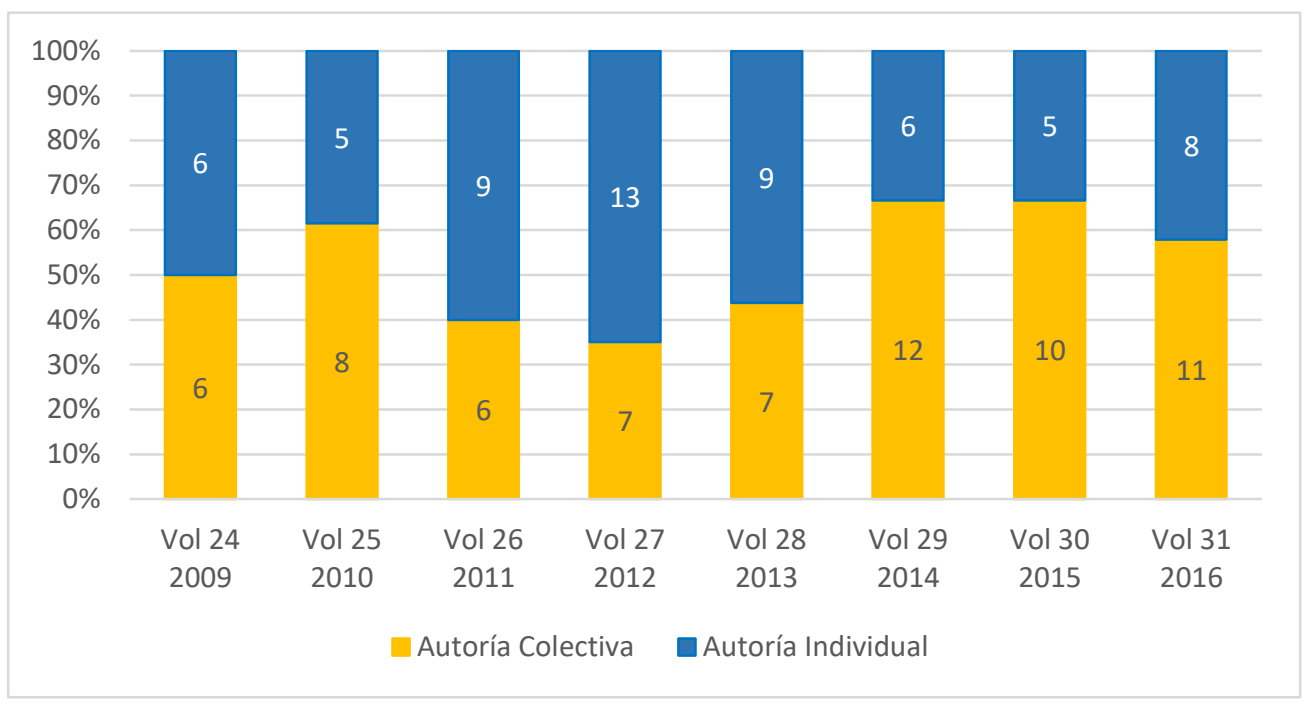

Fuente: Elaboración propia

Desagregando esta información para cada año resulta imposible plantear una tendencia global, pues se observa que los artículos de autoría colectiva superaron a los de autoría individual en cuatro años: en 2010 (volumen 25 de la revista), cuando la proporción fue de 3 a 2, y consecutivamente en 2014, 2015 y 2016 (volumen 29, 30 y 31 de la revista), cuando esa proporción fue de 2 a 1 y nuevamente 3 a 2 en los dos últimos años. Esto puede sugerir una nueva tendencia a reafirmar en las futuras ediciones respecto de una mayor co-autoría en la producción de artículos originales, lo que se condice en lo expuesto por Zamora-Bonilla y González sobre la colaboración científica, particularmente en atención a la "naturaleza de la empresa" por referencia a los 
beneficios que produce esta práctica "de cara a una distribución apropiada del esfuerzo de investigación y de los derechos de propiedad" (Zamora-Bonilla y González, 2014). Esto aun cuando a veces se lo interprete como una suerte de "capitalismo académico" que no más pretende "sacar" a los profesores e investigadores de la quietud y tranquilidad que supone una forma tradicional de organización (Fernández Rodrigo, 2011) como es la universitaria. Sólo a inicio y mitad del período bajo estudio los artículos de autoría individual empatan y/o superan a los de autoría colectiva, estableciéndose cierta supremacía de lo colectivo en el último trienio. Realizado el recuento de firmas, la información obtenida es la que consigna la Tabla $n^{\circ} 2$.

Tabla n²: Cantidad y porcentaje de autores (2009-2016)

\begin{tabular}{|c|c|c|c|c|}
\hline Firmas & Artículos & \% Acumulado & Firmas & \% Acumulado \\
\hline 1 & 63 & 49,2 & 63 & 26,5 \\
\hline 2 & 32 & 25,0 & 64 & 26,9 \\
\hline 3 & 25 & 19,5 & 75 & 31,5 \\
\hline 4 & 4 & 3,1 & 16 & 6,7 \\
\hline 5 & 4 & 3,1 & 20 & 8,4 \\
\hline Índice & $\mathbf{1 2 8}$ & $\mathbf{1 0 0}$ & $\mathbf{2 3 8}$ & $\mathbf{1 0 0}$ \\
\hline
\end{tabular}

Fuente: Elaboración propia

\subsection{Internacionalización de la colaboración}

Hemos decidido denominar "nacionalidad de la autoría" a la clasificación de la adscripción institucional de los autores en función del país en que se encuentran. Así, podemos distinguir entre "autoría nacional" para los artículos cuyos autores declaran adscripción a instituciones chilenas y "autoría extranjera", para los artículos cuyos autores forman parte de instituciones de otros países. Constatamos la autoría extranjera es levemente mayoritaria, con un $51 \%$ (121 autores), mientras que la nacional llega al 49\% (117 autores). El país con mayor presencia, después de Chile, fue España, con 38 autores (15\%), le siguen Argentina, con 25 (10\%), México, con $13(5 \%)$ y Colombia, con 10 autores (4\%) (Ver Gráfico n²).

Gráfico n²: Comparación Colaboración Internacional (2009-2016)

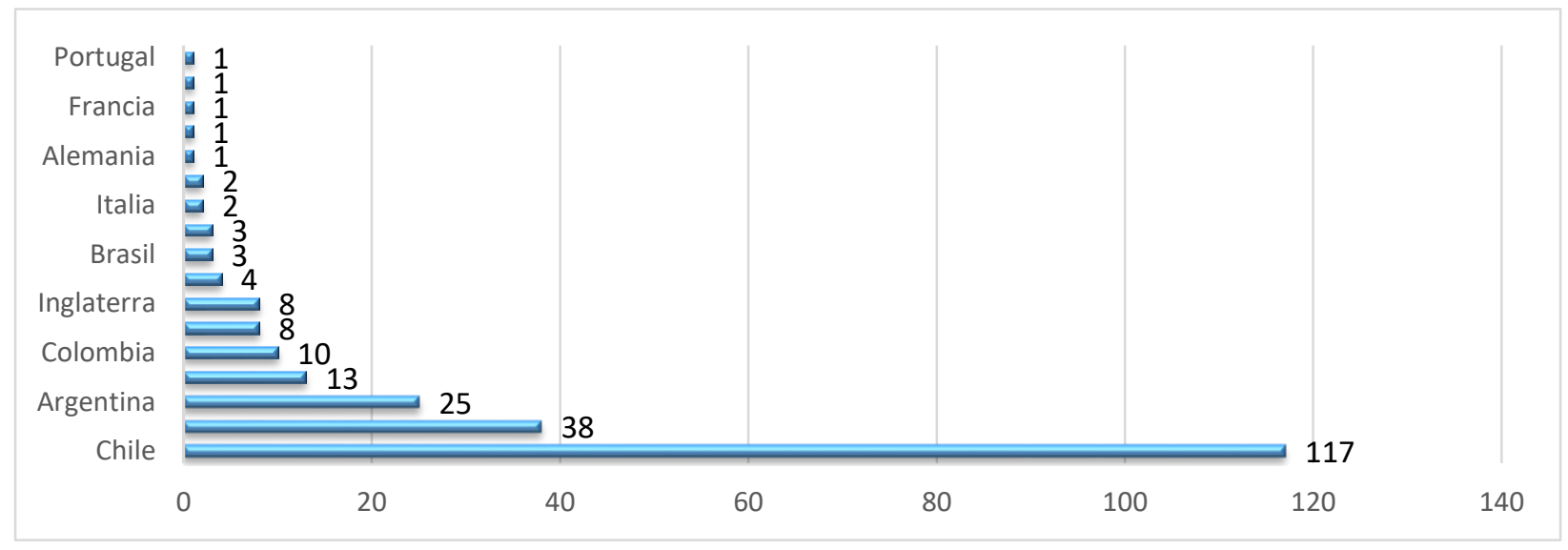

Fuente: Elaboración propia

En este punto, y según los datos indicados, parece que Revista INVI ha apostado por alejarse del patrón endogámico de la ciencia nacional (Gómez Morales, 2012) a lo largo del período bajo estudio, y que su operación editorial parece acercarse a los propósitos de "mejorar la calidad de los manuscritos seleccionados, conseguir un mayor flujo de manuscritos especializados de corriente principal, o de cobertura geográfica más global, o de temas emergentes que incluyan autores con redes científicas internacionales". (Santoro et.al, 2013) No obstante, la información disponible nos permite observar que en el caso de Revista INVI se ha producido una 
creciente colaboración internacional en la producción científica, por cuanto al observar los artículos que han sido producidos por investigadores de varios países, por ejemplo, en el portal de SCImago Journal \& Country Rank, constatamos importantes variaciones en el transcurso del período en análisis.

\subsubsection{Evolución de la autoría internacional en SCImago}

Según la información proporcionada por el portal de SCImago (Gráfico n³), la colaboración internacional en la autoría de artículos científicos ha experimentado cierta irregularidad en el ámbito de los estudios territoriales. Los indicadores en este factor son Vol 24(2009): 6.25; Vol 25(2010): 17.65; Vol 26(2011): 5,0; Vol 27(2012): 21,74, Vol 28(2013): 21.43; Vol 29 (2014) 20,00 y Vol 31(2015): 12,50. Estimamos que este último descenso va a repuntar en el último volumen, una vez que SCImago Journal \& Country Rank procese el año 2016. No obstante, en esta baja en el índice de colaboración internacional hacia el final del período, debe ser matizada con la situación experimentada por los autores de nacionalidad argentina y española que han experimentado un aumento explosivo en la publicación de manuscritos.

\section{Gráfico n`3: Colaboración Internacional en SCImago (2009-2016)}

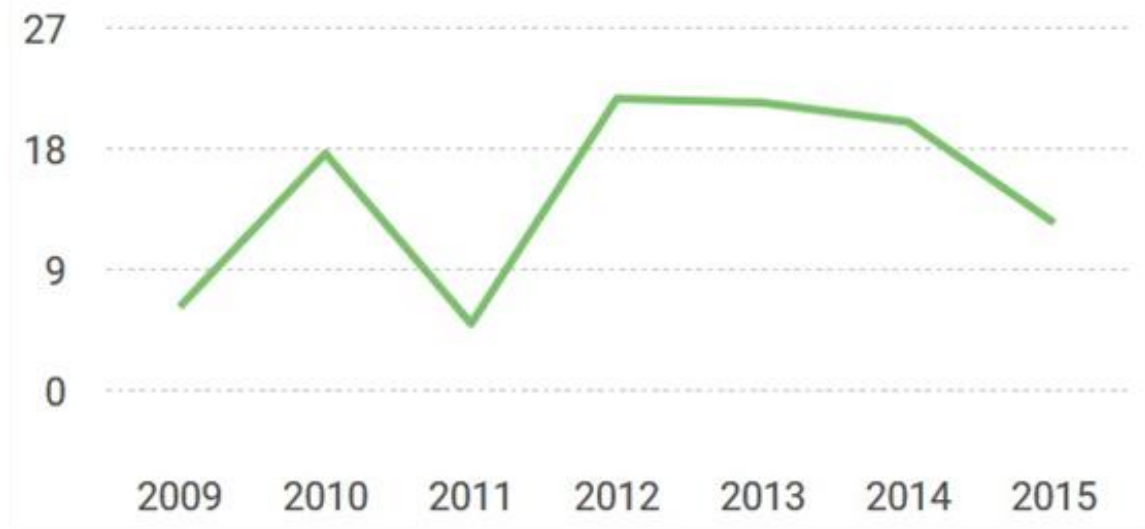

Fuente: SCImago.com

\subsection{Género}

Otro factor relevante de considerar es el que dice relación con el género de los autores. De acuerdo a las cifras de Conicyt, "Chile se encuentra por debajo de países como Colombia, Argentina, México y Brasil respecto al porcentaje de mujeres científicas que aparecen como autoras en publicaciones, y aquellos artículos cuyo autor o autores principales son mujeres, reciben menos citas si se les compara con autores hombres en la misma posición, lo que da cuenta de las dificultades que enfrentan las investigadoras" (El Mostrador, 2015). En virtud de lo anterior, el análisis del género de los autores de Revista INVI, calculado sobre el total de autores (238), permitió constatar que un $60 \%$ de los autores es de género masculino (142 autores), mientras que tan solo un $40 \%$ de las autoras es mujer (96 autoras). La equidad de género en la autoría es una constante asimétrica a lo largo de todo el período, con prominentes fluctuaciones, como se aprecia en el Gráfico n4, llegando a igualarse la presencia masculina/femenina en el año 2015, pero cuya brecha vuelve a abrirse al año siguiente. 
Gráfico n4: Evolución en el género de los autores (2009-2016)

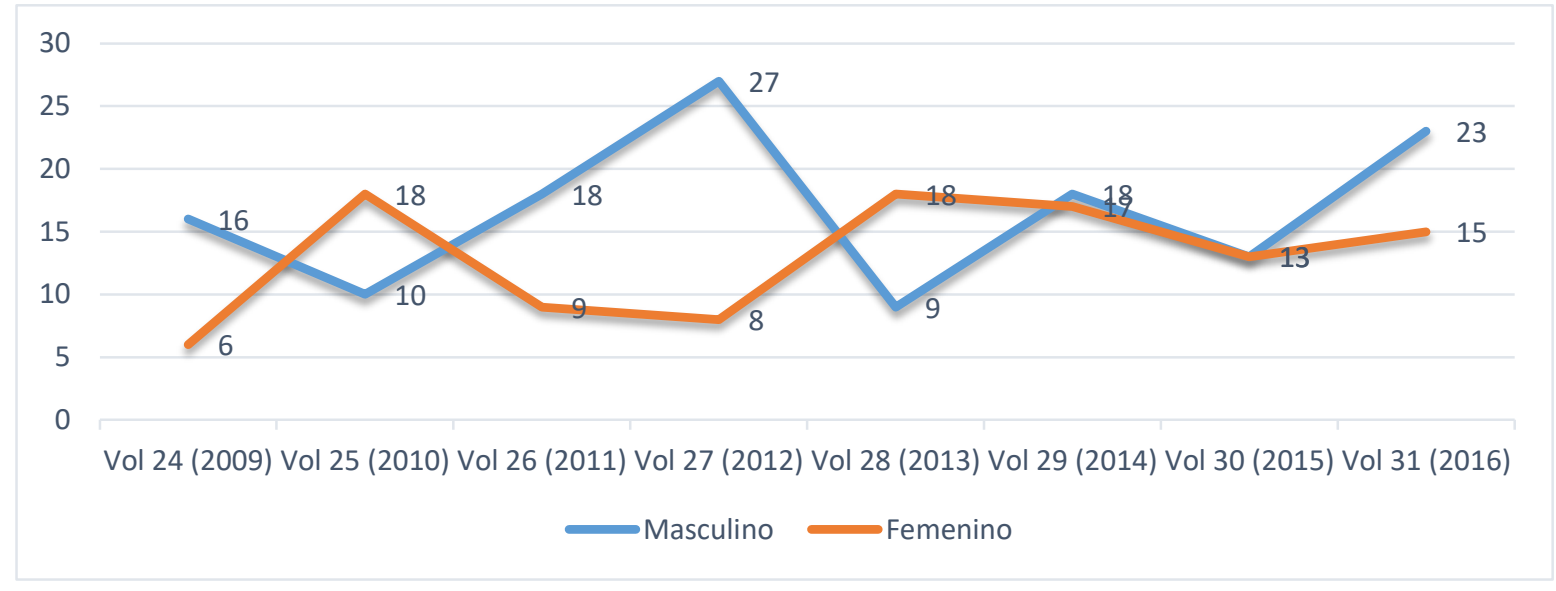

Fuente: Elaboración propia

\subsection{Profesión}

En cuanto a la profesión, claramente la arquitectura es mayoritaria (Tabla $n^{\circ} 3$ ), por cuanto es la disciplina de la que proviene el $44 \%$ de los autores, lo cual se condice perfectamente con la raigambre de la revista en su origen más puro, como es una publicación de arquitectura propiamente tal. Le sigue la geografía, con un $17 \%$ (40 autores), y, en tercer lugar, la sociología con un 16\% (39 autores). El resto de las profesiones se compone a grandes rasgos de la antropología con un 5\% (12 autores), trabajo social 4,2\% (10 autores), ciencias políticas, ingeniería y psicología (2,9\%; 7 autores cada una). Se constata con claridad una tendencia hacia el equilibrio en la participación de arquitectos frente a autores de otras disciplinas, con la sola excepción del Volumen 27 del 2012, donde la brecha entre estas categorías fue bastante amplia (7 versus 28).

Tabla n`3: Profesión de los autores (2009-2016)

\begin{tabular}{|c|c|c|c|c|c|c|c|c|c|}
\hline Profesión & $\begin{array}{c}\text { Vol 24 } \\
(2009)\end{array}$ & $\begin{array}{c}\text { Vol 25 } \\
(2010)\end{array}$ & $\begin{array}{c}\text { Vol 26 } \\
(2011)\end{array}$ & $\begin{array}{c}\text { Vol 27 } \\
(2012)\end{array}$ & $\begin{array}{c}\text { Vol 28 } \\
(2013)\end{array}$ & $\begin{array}{c}\text { Vol 29 } \\
(2014)\end{array}$ & $\begin{array}{c}\text { Vol 30 } \\
(2015)\end{array}$ & $\begin{array}{c}\text { Vol 31 } \\
(2016)\end{array}$ & TOTALES \\
\hline Arquitectura & 15 & 16 & 16 & 7 & 14 & 12 & 12 & 13 & 105 \\
\hline Otras Profesiones & 7 & 12 & 11 & 28 & 13 & 23 & 14 & 25 & 133 \\
\hline Totales & 22 & 28 & 27 & 35 & 27 & 35 & 26 & 38 & 238 \\
\hline
\end{tabular}

Fuente: Elaboración propia

\subsection{Referencias bibliográficas}

Este elemento es importante porque nos permite medir el grado de profundidad en la investigación, como bien lo señala Culebras-Fernández "las referencias a trabajos previos, simbolizan asociaciones conceptuales de ideas científicas reconocidas como provechosas por el autor que las cita” (Culebras Fernández et.al, 2008) o Pérez Borgues, respecto a que sirven para "determinar la exhaustividad con que se ha desarrollado un trabajo científico, para conocer cuán avanzado está un tema en el mundo, para identificar fuentes relacionadas o autores de impacto en una materia dada" (Pérez Borges, 2010). El análisis de las referencias bibliográficas indicó que, en todo el período y en los 128 artículos objeto de este estudio, se empleó 4.428 referencias, promediando 34,59 por artículo y 553,5 por volumen, siendo el mínimo de referencias citadas 5 y el máximo 90. Efectuados los cálculos el indicador de productividad de las referencias bibliográficas según el método adoptado (Pulgarín, Carapeto y Cobos, 2004), la media resultante es de 34,5 referencias bibliográficas en relación al número de artículos originales por volumen. De estos datos se desprende que el mayor índice de referencias (Gráfico n5) se obtuvo en el año 2016 (Vol. 31), con 43,5 y el menor en el año 2009 (Vol.24), 25,1. Por otra 
parte, el total de notas a pie de página es de 6.282, siendo el promedio por volumen de 785,25 notas a pie y por artículo de 49,07 con mínimo de 9 y máximo de 110.

\section{Gráfico n5: Referencias por artículos (2009- 2016)}

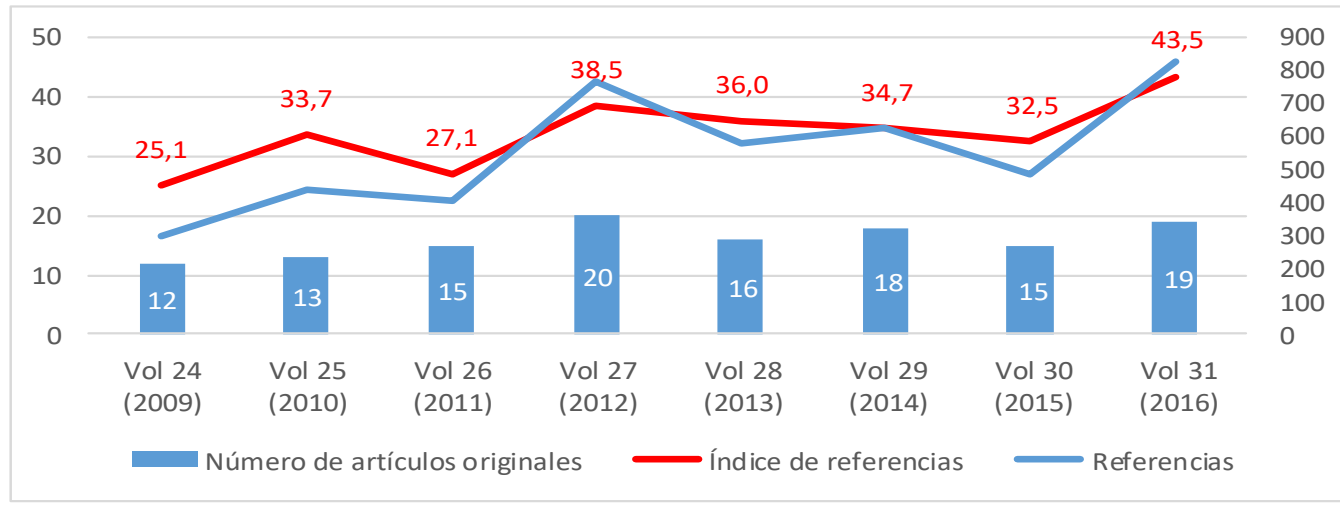

Fuente: Elaboración propia

\subsection{Descripción del estado actual de nuestros indicadores de impacto}

Revisados los indicadores proporcionados por los grandes consorcios, hay varias consideraciones. En primer lugar, en SciELO, llama la atención que los cinco artículos originales más visitados corresponden a un artículo de autor español: "Calidad de vida y medio ambiente urbano: indicadores locales de sostenibilidad y calidad de vida urbana", (2009: Hernández Aja, A.), y de los cuatro siguientes, tres autores colombianos y uno de nacionalidad peruana. Recién en sexto y séptimo y lugar aparecen artículos de autores chilenos. Este hecho refrenda aquel mayor grado de internacionalización respecto de la autoría de los artículos, sin embargo, recordemos que el $49 \%$ de los autores (117), es decir casi la mitad, son chilenos. Este dato constituye una pista interesante para formular interrogantes respecto de las potenciales comunidades o redes de lectores de la Revista (¿cuáles son las redes de lectores de la revista?). Similar interrogante nos plantea Redalyc, ya que sus datos acerca de los accesos nos indican que, de un número de 46.000 descargas, éstas se realizan mayormente en México y EE.UU, lo que puede sugerir que en ciertas zonas geográficas hay usos mayoritarios de portales con los que la población está más familiarizada idiomárticamente (Gráfico nº6).

Gráfico $n^{\circ}$ 6. Redalyc: Descargas por país

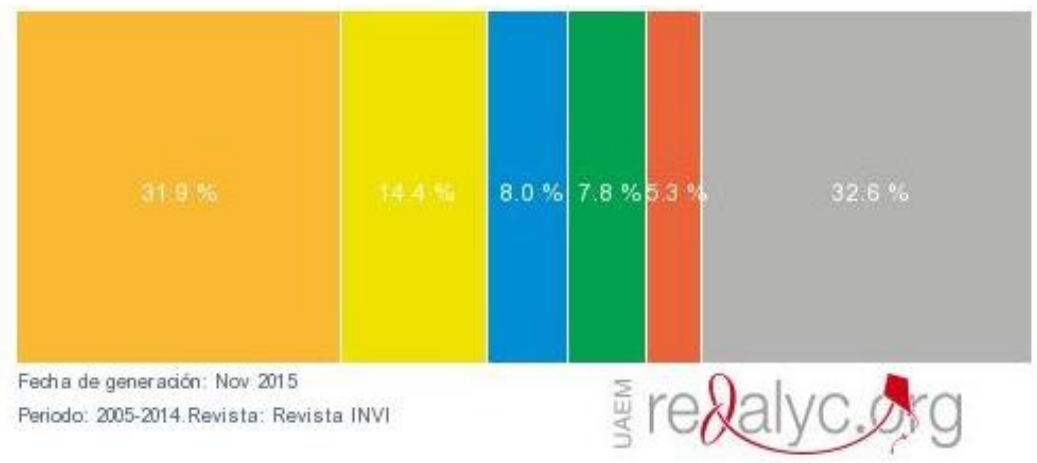

México Estados Unidos $\square$ Canada Chile $\square$ España otros 


\subsection{Factor de impacto/Cantidad de Citas}

El factor de impacto según el último procesamiento correspondiente a diciembre 2016 en esta base de datos es de 0,2963 para un período de tres años y de 0,2703 para uno de dos, los cuales son mejores que los de las publicaciones comparables dentro del país y dentro del mismo ámbito de estudio, tales como Revista Eure $(0,009$ y 0,000 respectivamente) y Revista Nortegrande $(0,2549$ y 0,2059$)$. Este dato no es menor si se tiene en cuenta que estas últimas poseen estándar WOS.

Revisando el resto del panorama actual en cuanto al nivel de citas recibidas mediante la información disponible en cada una de las fuentes, esto es, WOS, SCOPUS, SCImago y SciELO, tenemos diferentes escenarios, pero que convergen en una misma dirección y que dice relación con una leve baja en el año 2015 respecto de la línea de ascenso que se venía manteniendo hasta el 2014, factor que debe ser sometido a evaluación con el propósito de tomar las remediales que sean pertinentes (Gráfico $\mathrm{n}^{\circ} 7$ ).

Gráfico n7: Nivel de citas que registran los grandes consorcios (2009-2016)

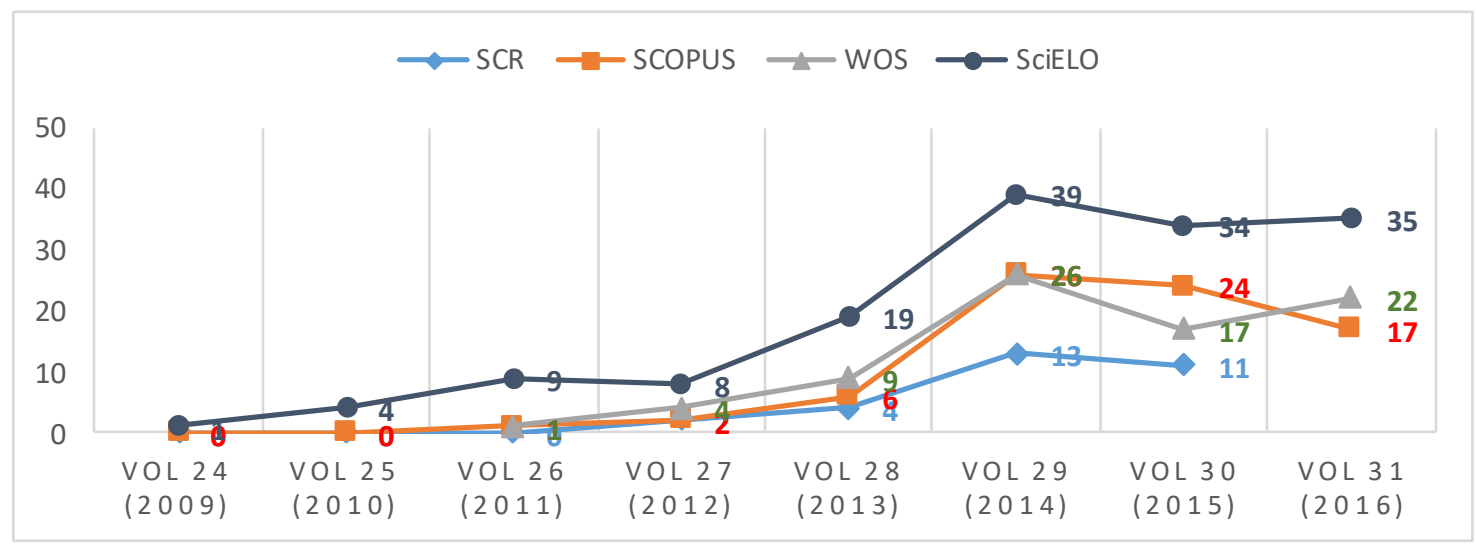

Fuente: Elaboración Propia con base a la información on-line de cada uno de los consorcios

En la Web Of Science, se promedia para el período bajo estudio una cifra de 13,17 de citas por año, con 79 citas en total. Los artículos más citados, de acuerdo al h-index 4 de esta revista, han sido: Urbanismo proempresarial en Chile: políticas y planificación de la producción residencial en altura en el pericentro del gran Santiago (2012: López-Morales, E., Gasic, I. y Meza, A.); en segundo lugar, Exclusión y desigualdad espacial: retrato desde la movilidad cotidiana (2010: Jirón M, Paola; Lange V, Carlos; Bertrand S, María), en tercer término: El proceso de reconstrucción de viviendas en el centro de Talca (2013: Rasse, A. y Letelier, F.) y en cuarto lugar, Más que una suma de casas: la unidad vecinal villa San Pedro de Coronel (2009: Demiddel, Stéphane Franck; Pérez Bustamante, Leonel).

En SCOPUS, la Revista totaliza 76 citas en el período, promediando 15,2 citas por año, y en donde los artículos más citados, de acuerdo al h-index 4, son: Climate change and Urban climate: Relations between thermal zones and the socioeconomic conditions of the population of Santiago de Chile (2010: Romero, H., Salgado, M., Smith, P.); Distinción social y hábitat residencial en américa Latina (2011: Roitman, S.); Vivienda social en Santiago de Chile. Análisis de su comportamiento locacional, período 1980-2002 (2012: Tapia, R.) y El proceso de reconstrucción de viviendas en el centro de Talca (2013: Rasse, A. y Letelier, F.).

En SciELO, además de los accesos a los números indicados, el número de citas recibidas es de 149 para una media de 5,61 artículos por fascículo. Aunque autocitándose en 33 oportunidades, el total de citas se distribuyen entre otras 56 revistas, pero lo relevante acá es que dos de éstas, y que precisamente le siguen en orden descendente de citas concedidas son precisamente sus "competidoras", como ya se ha dicho, o aquellas de su misma área u objeto de estudio, de reconocido prestigio tanto nacional como internacional. 
Grafico n`8. Mapa de calor con citaciones SciELO

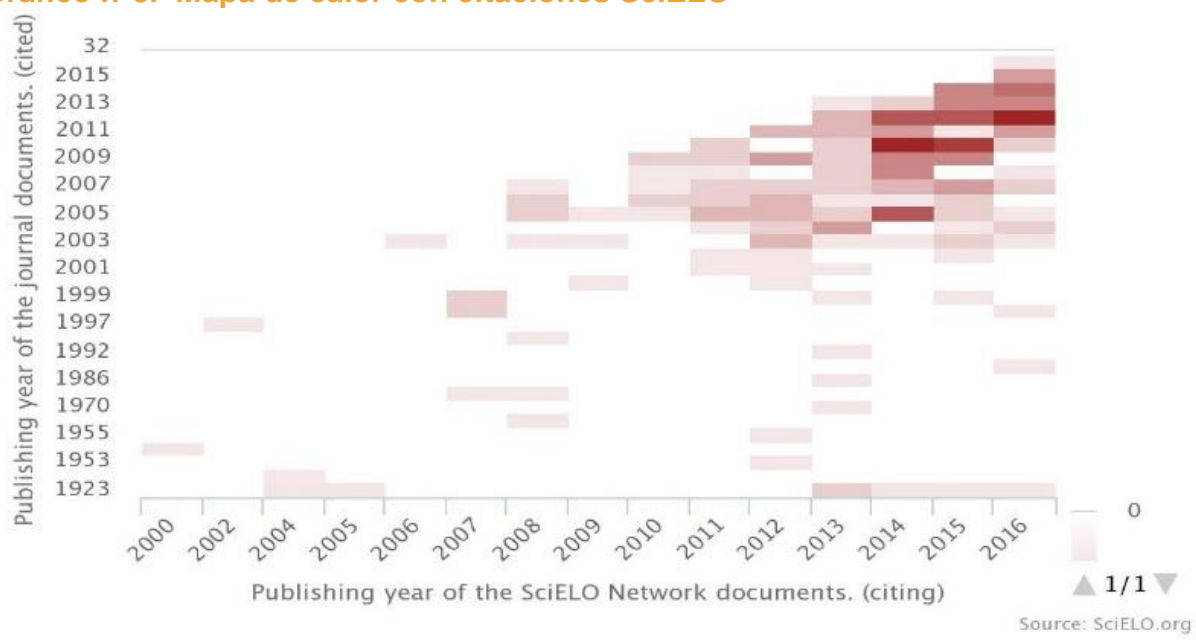

Fuente: SciELO.org

Por último, cabe resaltar lo ya señalado que tanto para WOS como SCOPUS, el H-Index es 4, para SCImago 3. Esto quiere decir que, para los grandes consorcios, cuatro artículos de los 128, es decir, 3,125\% al menos tienen cuatro citas. Sin embargo, SciELO, que para estos efectos emplea datos obtenidos a partir de Google Scholar, obtiene cifras más auspiciosas (Gráfico n8), mientras que para Journal Scholar Metrics este indicador es 7.

\subsection{Palabras Clave}

En los 128 artículos considerados fueron empleadas 504 palabras clave, cada una de las cuales fue aportada por los propios autores a fin de reflejar la naturaleza de su texto. Las palabras clave, en consecuencia, constituyen una información valiosa que, no estando sujeta a la intervención de un analista, entrega un reflejo de "la red de relaciones conceptuales" (Ecured, 2016) de la revista, elemento esencial y que le otorga sentido a su existencia como publicación de corriente principal. Bien vale la pena rescatar lo señalado por José Ignacio De Granda Orive, en cuanto a que "las palabras clave no sólo son útiles para realizar una búsqueda, sino que además sirven para analizar los trabajos por la materia estudiada, permitiendo así descubrir la evolución de las corrientes investigadoras y los aspectos que más o menos interesan a los investigadores" (Granda Orive, 2003).

En el período abordado, este aspecto puede medirse desde un punto de vista tanto "puramente" métrico como "esencialmente" semántico. En cuanto a aquél, puede establecerse que un $99 \%$ de éstos emplea dos descriptores de materia, un $96 \%$ lo hace con tres y el $100 \%$ emplea uno a lo menos, cabe hacer notar que un $63,2 \%$ de los artículos requiere emplear a lo menos cuatro descriptores para reflejar la naturaleza de su contenido y un 32\% cinco, arrojando una media de 3,93 palabras clave en el período, con una mínima de 1 y una máxima de 8, el artículo que más keywords empleó: Estigmatización territorial en acción (2014: Loïc J.D. Wacquant). Sólo un 2,3\% de los artículos emplea 6 o más palabras clave. A nivel de contenido, las palabras que se repiten con mayor frecuencia son Vivienda (42), Social (28), Urbano/a (33), Política/s (25), Ciudad (16), Hábitat y Chile $(10 \mathrm{c} / \mathrm{u})$. Una representación gráfica bastante similar de esta frecuencia es la que se puede ver en el home de Redalyc y que se ilustra a continuación: 


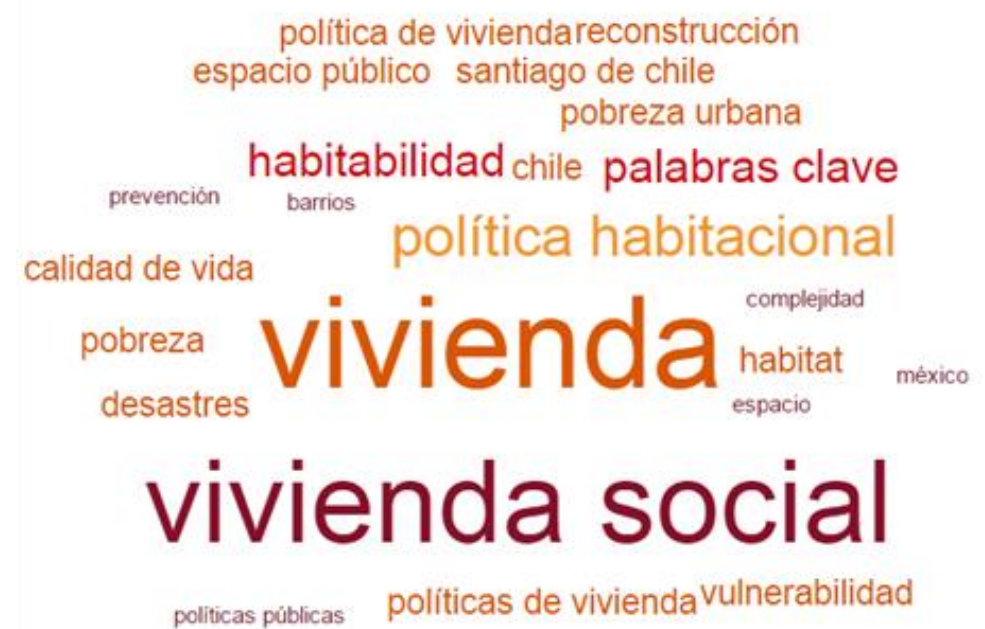

Fuente: Redalyc.org

\section{Discusión/Conclusiones}

A continuación, discutiremos tres aspectos centrales obtenidas de nuestro análisis: a) el carácter general de este estudio; b) el indicador de colaboración obtenido y; c) la información sociodemográfica generada.

\subsection{Carácter general del estudio}

El estudio bibliométrico aquí propuesto constituye una iniciativa pionera en el área de los estudios territoriales en América Latina, ya que hasta el momento no se conoce un análisis pormenorizado de indicadores de actividad de revistas especializadas en dicha materia, lo que otorga valor y relevancia al trabajo propuesto, pero que, al mismo tiempo, hace que dicho valor sea relativo por cuanto se carece de referencias para establecer puntos de comparación y criterios de evaluación pertinentes. En este sentido, la situación que enfrentamos es aún más compleja que la descrita por Camps (Camps, 2007).

En efecto, dicho autor plantea que son pocos los estudios de bibliometría que se enfocan en el análisis de los artículos originales, lo que hace difícil establecer comparaciones entre publicaciones y, así, estimar tendencias generales en un campo de estudios. En el caso de Revista INVI esto es justamente así: las tendencias obtenidas no son posibles de cotejar con otras publicaciones similares, por cuanto no existen estudios que lo permitan. Este punto no es menor si se considera que la información bibliométrica es fundamental para estimar las características, cambios y tensiones en la producción científica contemporánea.

Por otra parte, existe otro tipo de información que sí es más abundante, como es la provista por redes como SciELO o WOS (Web of Science, ex-ISI). Sin embargo, tal información, si bien posibilita establecer jerarquías de indexación y asignar valores diferenciales a la productividad científica de un autor, no permite hacer estimaciones como las propuestas en este artículo. En la perspectiva de avanzar en una comprensión más acabada y detallada de la productividad científica en el campo de los estudios territoriales parece imprescindible relacionar ambos tipos de información. Además, no parece adecuado desestimar las voces críticas que han surgido respecto de la indexación de las revistas y la subsecuente generación de indicadores para la gestión de instituciones académicas (Villalobos, 2013). 


\subsection{El indicador de colaboración}

Un segundo aspecto relevante tiene que ver con la colaboración entre autores. El índice de colaboración o productividad obtenido es de 1,83, el que resulta bajo en comparación con el obtenido por Camps (2007) para la revista Universitas Médica, que es de 3,32. O en comparación con el obtenido por Paganini y Raiher (2006), para la OPS/OMS que es de 2,7. O en comparación con el obtenido por Escorcia-Otálora y Poutou-Piñales, (2008) para la revista Universitas Scientiarum, que es de 2,95.

Este indicador es relevante puesto que permite estimar la situación de la Revista INVI, en relación a la tendencia general esperada, y deseada de acuerdo al discurso internacional e institucional actual, respecto de una productividad científica colectiva o en equipo, transdisciplinaria y en colaboración internacional (CONICYT, 2012). Un índice de colaboración o productividad de 1,83 señala que esta tendencia no se ha materializado en la Revista INVI, sino que ella se encuentra en una situación semejante a la mencionada para el caso de "áreas como el derecho, la filosofía y la historia”, donde predominan los trabajos sin colaboración (CONICYT, 2012). La salvedad se encuentra en que esa relativamente baja colaboración es, en muchos casos, de tipo interdisciplinar, pues involucra autores de la arquitectura que se vinculan a otros provenientes de otras disciplinas, factor demostrado con el análisis efectuado en la tabla $N^{\circ} 3$.

\subsection{Perfil sociodemográfico}

El rasgo recién mencionado nos permite discutir la información obtenida respecto del perfil sociodemográfico de los autores de Revista INVI. Según se mostró anteriormente, la participación masculina es casi idéntica a la femenina, bastante mejor que la detectada por CONICYT (2013) en su reporte sobre participación femenina en sus programas de desarrollo científico para el período 2001-2013, en Chile, donde detecta que sólo el 40,7\% de las postulaciones admisibles es de mujeres.

Otro aspecto del perfil sociodemográfico es la nacionalidad de los autores. Al respecto, la presencia de autores extranjeros se ha incrementado notablemente en los últimos años. Este es un aspecto valorable en la evolución de la revista. Sin embargo, esto no implica que se produzca colaboración internacional en la producción de artículos, tal como sugiere la tendencia actual, en la cual "sólo los trabajos en colaboración internacional e internacional+nacional alcanzan impactos sobre la media del mundo" (CONICYT, 2012). Es más, este tipo de colaboración es, segun nuestro análisis, inexistente.

Por último, respecto del perfil sociodemográfico también constatamos la mayoritaria presencia de autores provenientes de la disciplina de la arquitectura (44\%). No obstante, esta situación se ha ido modificando progresivamente, para llegar a una situación actual (año 2015 y 2016) donde ese predominio se ve claramente equiparado por la presencia autores de las ciencias sociales (principalmente de la geografía y la sociología) en misma cantidad de representantes.

La evolución en el período de tiempo de estos indicadores nos señala que existe una tendencia clara hacia la internacionalización de la autoría y la igualdad de género de los autores, ya que en ambos casos constatamos un comportamiento homogéneo y muy similar, con muy pocas brechas, especialmente en este último aspecto en el cual durante los últimos tres años los indicadores prácticamente se equiparan, lo que se condice con la aparición de nuevos estudios enfocados en reconocer la presencia igualitaria de género en la autoría y que el porcentaje de mujeres y porcentaje de hombres autores de documentos en cada revista revelaría ciertas situaciones de desigualdad (Mauleón et.al, 2013) no presentes en absoluto en el caso de Revista INVI. 


\section{Bibliografía}

Aguado-López, Eduardo, Rogel-Salazar, Rosario, Garduño-Oropeza, Gustavo, Becerril-García, Arianna, Zúñiga-Roca, María Fernanda y Velázquez-Álvarez, Alejandro (2009). Patrones de colaboración científica. A partir de redes de coautoría. En: Convergencia Revista de Ciencias Sociales n 16. http://www.redalyc. org/articulo.oa? id=10512244010. Consultado el 9 de diciembre de 2013

Camps, Diego (2007). Estudio bibliométrico general de colaboración y consumo de la información, en artículos originales de la revista Universitas Médica, período 2002 a 2006. En: UNIVERSITAS MÉDICA 2007 VOL. 48 № 4.

Comisión nacional de ciencia y tecnología de Chile, CONICYT (2012). Principales indicadores bibliométricos de la actividad científica chilena 2010. Informe 2012.

http://www.cincel.cl/Documentos/Recursos/Principales indicadores bibliometricos actividad cientifica chilena Informe 2012. pdf

Comisión nacional de ciencia y tecnología de Chile, CONICYT (2013). Reporte Participación Femenina en Programas CONICYT 2013. http://www.conicyt.cl/wp-content/uploads/2013/05/Participacion Femenina sem2 2013.pd:

Culebras-Fernández, J. et al. ¡Cuidado!, sus referencias bibliográficas pueden ser estudiadas. En: Nutr. Hosp., Madrid, v. 23 , n. 2, abr. 2008. Disponible en http://scielo.isciii.es/scielo.php?script=sci arttext\&pid=S0212

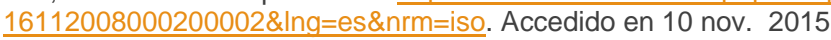

Díaz Mujica, Desireé. (2010) Análisis bibliométrico de la revista Anales Venezolanos de Nutrición. En: An Venez Nutr, Caracas, v. 23, n. 1, jun. 2010. Disponible en http://www.scielo.org.ve/scielo.php?script=sci arttext\&pid=S0798$07522010000100006 \& \operatorname{lng}=e s \& n r m=i s 0$. Consultado el 4 de diciembre de. 2014.

Escorcia-Otálora, Tatiana y Poutou-Piñales, Raúl (2008). Análisis bibliométrico de los artículos originales publicados en la revista Universitas Scientiarum (1987-2007). En: UNIVERSITAS SCIENTIARUM Disponible en línea en: http://www.javeriana.edu.co/universitas scientiarum 2008, Vol. 13 №3, 236-244

Fernández Albornoz, Rodrigo. Capitalismo académico en la Educación Superior Chilena ¿Modelo exitoso para el desarrollo de la ciencia? Análisis de las tendencias en la productividad científica en investigación durante la última década. En: Science for Innovation Año 1- № 1, octubre 2011, Págs. 36 - 45

García Ramón, María Dolores, Belii, Mireia, Clos, Isabel. (1988). La receptividad a influencias extranjeras en revistas españolas de geografía. Un análisis bibliométrico. En: Anales de geografía de la Universidad Complutense, ISSN 0211-9803, № 8, 1988, págs. 11-24. http://dialnet.unirioja.es/servlet/articulo?codigo=86313. Consultado el 9 de diciembre de 2013.

Gómez Morales, Yuri Jack; Jaraba-Barrios, Bruno; Guerrero-Castro, Javier \& López-López, Wilson. Entre Internacionalización y Consolidación de Comunidades Académicas Locales. Sobre la Revista Latinoamericana de Psicología. En: Revista Colombiana de Psicología vol. 21 n.o 1 enero-junio 2012, ISSN 0121-5469, Bogotá, Colombia, pp. 97-110.

Granda Orive, José Ignacio de; García rio, Francisco y Callol Sánchez, Luis. Importancia de las palabras clave en las búsquedas bibliográficas. En: Rev. Esp. Salud Pública [online]. 2003, vol.77, n.6 [citado 2016-01-06], pp. 765-767. Disponible en: http://scielo.isciii.es/scielo.php?script=sci arttext\&pid=S1135-57272003000600010\&lng=es\&nrm=iso. ISSN 1135-5727

Jiménez-Contreras, Evaristo. (2000) Los métodos bibliométricos. Estado de la cuestión y aplicaciones. En: Cuadernos de documentación multimedia. №10.

http://pendientedemigracion.ucm.es/info/multidoc/multidoc/revista/num10/paginas/pdfs/ejcontreras.pdf. Consultado el 9 de diciembre de 2013.

López, A., Núñez, C., Vicente-Herrero, M., Monroy, N., Sarasibar, H., Tejedo, E. Análisis bibliométrico de la productividad científica de los artículos publicados por diferentes revistas españolas entre los años 1997 y 2006 . En: Medicina BalearVol.23, núm. 1, 2008. http://dialnet.unirioja.es/descarga/articulo/2652220.pdf. Consultado el 2 de diciembre de 2014.

Mauleón, E., Hillán, L., Moreno, L., Gómez, I., \& Bordons, M. (2013). Assessing gender balance among journal authors and editorial board members. En: Scientometrics, 95(1), 87-114.

Paganini, J. M., \& Rahier, S. (2006). Análisis bibliométrico de las publicaciones sobre investigación en servicios de salud: tendencias y características. Facultad de Ciencias Médicas de la Universidad Nacional de La Plata y Centro Interdisciplinario Universitario para la Salud. Recuperado de http://www.inus.org.ar/documentacion/Documentos\% 20Tecnicos/Analisis bibliometrico publicaciones investigacion.pdf.

Pérez Borges, Aylén. Empleo de citas y referencias bibliográficas en trabajos científicos. Documentación de las Ciencias de la Información. 2010, vol. 33, 185-193.

Pulgarín, A., Carapeto, C., \& Cobos, J.M. (2004). Análisis bibliométrico de la literatura científica publicada en Ciencia. Revista hispano-americana de ciencias puras y aplicadas (1940-1974). En: Information Research, 9(4) paper 193 Available at http://InformationR.net/ir/9-4/paper193.htm 
Santoro, Calogero M, Standen, Vivien G, Angelo, Dante, \& Gavilán, Vivian. (2013). Internacionalización de Revistas Científicas en Campos Emergentes como Antropología: Desafíos y Oportunidades para Chungara. En: Chungará (Arica), 45(3), 367-369. Recuperado en 05 de enero de 2016, de http://www.scielo.cl/scielo.php?script=sci arttext\&pid=S071773562013000300001\&lng=es\&tlng=es. 10.4067/S0717-73562013000300001.

Tomás-Casterá, Vicente, Sanz-Valero, Javier, y Wanden-Berghe, Carmina. (2010). Estudio bibliométrico de la producción científica y uso de la revista chilena de nutrición a través de la red scielo (2002 a 2007). En: Revista chilena de nutrición, 37(3), 330-339. http://www.scielo.cl/scielo.php?script=sci arttext\&pid=S0717-75182010000300008\&lng=es\&tlng=es. Consultado el 2 de diciembre de 2014

Villalobos, S. (2013). Revistas indexadas y otros vicios académicos. En: Revista Chilena de Literatura, 0(84). Recuperado de http://www.revistaliteratura.uchile.cl/index.php/RCL/article/view/28509/30279

Zamora-Bonilla, J.; González de Prado Salas, J. (2014). Un análisis inferencialista de la co-autoría de artículos científicos. En: Revista Española de Documentación Científica, 37(4): e064. doi: http://dx.doi.org/10.3989/redc.2014.4.1145 


\section{Datos de los autores}

\section{Luis Campos Medina}

Sociólogo (Universidad de Chile, Santiago, Chile). Doctor en Sociología (École des hautes études en sciences sociales, París, Francia), Académico del Instituto de la Vivienda de la Universidad de Chile. luiscampos@uchilefau.cl

\section{César Orellana Mejías}

Bibliotecario Documentalista (Universidad Tecnológica Metropolitana, Santiago, Chile) perteneciente al Centro de Documentación Edwin Haramoto Nishikimoto del Instituto de la Vivienda de la Universidad de Chile. cesarorellana@uchilefau.cl

\section{Gustavo Carrasco Pérez}

Arquitecto D.P.L.G (École Nationale Supérieure des Beaux Arts, Paris, Francia), Arquitecto (Universidad de Chile, Santiago, Chile). Académico del Instituto de la Vivienda de la Facultad de Arquitectura y Urbanismo de la Universidad de Chile. Arquitecto Jefe Unidad de Patrimonio Dirección de Obras Municipales de Santiago. gcarrasco@uchilefau.cl

Recibido - Received: 2016-08-03

Aceptado - Accepted: 2017-03-20

Este trabajo se desarrolló en el marco del Proyecto de Atracción de Capital Humano Avanzado denominado "De la vivienda al hábitat residencial desde la perspectiva del habitante" (PAI 79112022), financiado por CONICYT. Parte de sus resultados han sido presentados en el "3er Congreso ALTEHA de Teoría del Habitar" desarrollado en la Facultad de Arquitectura, Universidad de la República, en Montevideo-Uruguay el 19, 20 y 21 de junio de 2013 y en la "XIX Conferencia Internacional de Bibliotecología: Cómo innovar y gestionar la información en el Siglo XXI", realizada por el Colegio de Bibliotecarios de Chile, en Santiago de Chile, los días 3 y 4 de noviembre 2015.

\section{$(\mathrm{cc}) \mathrm{BY}$}

This work is licensed under a Creative Commons Attribution 4.0 United States License.

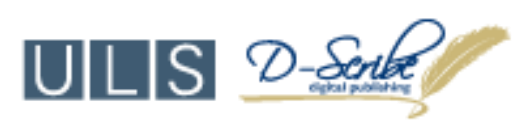

This journal is published by the University Library System of the University of Pittsburgh as part of its D-Scribe Digital Publishing Program and is cosponsored by the University of Pittsburgh Press. 